Cahiers d'études italiennes

18 | 2014

NOVECENTO... E DINTORNI

Da Torino a Parigi: Laura Malvano storica e critica d'arte

\title{
Filippo Baldinucci e le sue fonti francesi
}

Filippo Baldinucci et ses sources françaises

Filippo Baldinucci and his French Sources

\section{Evelina Borea Previtali}

\section{OpenEdition}

\section{Journals}

Edizione digitale

URL: http://journals.openedition.org/cei/1763

DOI: $10.4000 /$ cei. 1763

ISSN: 2260-779X

\section{Editore}

UGA Éditions/Université Grenoble Alpes

\section{Edizione cartacea}

Data di pubblicazione: 30 mars 2014

Paginazione: 149-153

ISBN: 978-2-84310-268-4

ISSN: 1770-9571

\section{Notizia bibliografica digitale}

Evelina Borea Previtali, «Filippo Baldinucci e le sue fonti francesi», Cahiers d'études italiennes [Online] 18 | 2014, online dal 30 septembre 2015, consultato il 26 mars 2021. URL: http:// journals.openedition.org/cei/1763; DOI: https://doi.org/10.4000/cei.1763 


\title{
FILIPPO BALDINUCCI E LE SUE FONTI FRANCESI
}

\author{
Evelina Borea Previtali
}

Lavorando nel 20 ir per l'edizione critica del libro Cominciamento e progresso dell'arte dell'intagliare in rame di Filippo Baldinucci, Firenze, I686 ${ }^{\mathrm{I}}$, ho pensato di stralciare da mie pagine già abbozzate il testo da dedicare a Laura. Il libro è il primo testo, consistente in un proemio e diciotto biografie (fra le quali tre di incisori francesi, Jacques Callot, Robert Nanteuil, François Spierre) che intende narrare la storia dell'incisione sino a quel momento: da Dürer a Rembrandt. Un libro noto, ma mai letto sinora a fondo.

Facendo l'edizione critica di un testo antico si cercano anzitutto le sue fonti. Così mi sono accorta che fra i libri consultati dal Baldinucci per raccogliere notizie e dati, oltre Vasari e Van Mander, Cornelis de Bie e Sandrart e tutti gli altri, cose ben note, c'era anche, non dichiarato come fonte, ma lodato nel proemio come incisore e come illustratore dei tre libri del matematico Girard Desargues, un certo Anton Bos (p. viI), che, dopo qualche perplessità, riconoscevo come nome storpiato di Abraham Bosse, il celebre autore del primo trattato sulle tecniche delle stampe ${ }^{2}$, ma anche di altri importanti saggi.

Infatti, per il passo che inizia nel Cominciamento (p. III) con la menzione di «il tedesco», ossia Martin Schongauer, sino a quello sui Sadeler, Baldinucci ripete con poche varianti e aggiunte quanto scritto da Abraham Bosse $^{3}$. Questi a quel tempo aveva certamente letto in proposito il capitolo

I. F. Baldinucci, Cominciamento e progresso dell'arte dell'intagliare in rame, a cura di E. Borea, Torino, Einaudi, 2013.

2. A. Bosse, Traicté des manières de graver en taille douce sur l'airin par le moyen des eaux fortes \& des vernix durs \& mols. Ensemble de la façon d'en imprimer les Planches \& d'en Construire la Presse, \& autres choses concernant les dits Arts, Paris, I645.

3. A. Bosse, Sentimens sur la distinction des diverses manières de peinture, dessein et graveure, et des originaux d'avec leurs copies, Parigi, I649, ristampato in ID., Le peintre converty aux précises et universelles règles de son art; Sentiments sur la distinction des diverses manières de peinture, dessins et gravure, textes réunis et présentés par Roger Armand Weigert, Paris, Hermann, I964, pp. I07-I85, in particolare pp. I6I-I64. 
vasariano su Marcantonio e gli altri intagliatori, di cui sintetizzando tiene conto, ma seguitando il suo racconto arriva oltre il tempo del Vasari, sino ai Sadeler e a Jan Saenredam, ai quali accennava il Van Mander nel I604, nel suo Schilderboeck, e a Francesco Villamena, cui aveva dedicato una breve biografia il Baglione nel I642. La riprova che Baldinucci si rifa al francese è nella sequenza non identica ma simile dei nomi di incisori tardocinquecenteschi ricordati, Cornelis Cort, Martino Rota Agostino Carracci, Cherubino Alberti, Francesco Villamena (con la stessa menzione della sua stampa da Paolo Veronese raffigurante La presentazione di Gesù al tempio), Jan e Raphael Sadeler (con la stessa citazione di Martin de Vos come loro principale ispiratore), Gilles Sadeler, Jan Saenredam, Hermann Müller, Willem Swanenburg (con la stessa menzione della tenerezza, ossia tendresse, delle sue stampe tratte da invenzioni di Rubens e di Abraham Bloemaert). Ma soprattutto è da notarsi la dipendenza del Baldinucci dal Bosse nella citazione en passant di quel Silvestro da Ravenna incisore, il cui nome ignoto al Vasari compare in Sentimens, qui credo per la prima volta, e che correrà presso i cultori di stampe sino a quando Giovanni Gori Gandellini nel I77I, con buoni argomenti, lo cancellò dalla storia come persona mai esistita ${ }^{4}$.

Questo nome, Silvestro da Ravenna, compare in verità anche nel libro del 1666 di Michel de Marolles, che gli attribuisce stampe in numero di ben settantaquattro', ciò spiegandosi con una erronea interpretazione delle stampe di area raffaellesca siglate con una $S$ e una $R$ intrecciate, poi variamente interpretate, a seconda se lette come SR o RS, Silvestro da Ravenna o sculptor ravegnanus, che sarebbe Marco Dente da Ravenna, oppure Raffaello Sanzio, che sarebbe l'indicazione di un'invenzione raffaellesca all'origine della stampa. Questione di lana caprina, viene da dire, tipica dei cultori non solo antichi del mondo iperspecialistico delle stampe; in ogni caso oggi, affinate l'indagine filologica e l'osservazione stilistica sul complesso di stampe prodotte nel primo cinquecento nell'ambito di Raffaello, nessuno mette in dubbio che l'autore che si nasconde dietro quella controversa sigla sia Marco Dente da Ravenna ${ }^{6}$.

D'altro canto il Baldinucci, anche se non lo dice, vede il catalogo del de Marolles come un vangelo, e forse è proprio dalla lettura di quello straordinario compendio ch'egli trae la voglia di scrivere il suo proprio libro,

4. G. Gori Gandellini, Notizie istoriche degli intagliatori, Siena, V. Pazzini e figli, I77I, vol. III, pp. I43-I44.

5. M. de Marolles, Catalogue de livres d'estampes et de figures en taille douce, avec un dénombrement des pièces qui y sont contenues, fait à Paris en l'année I666 par M. de Marolles, Paris, F. Léonard, I666, pp. 20, 37.

6. R. A. Weigert nel suo commento a A. Bosse, Le peintre Converty, cit., p. I62, scrive: «Silvestre, sans doute Meldolla lo Schiavone», ipotesi ovviamente non raccolta. 
si trattava infatti dell'unico testo nel quale, sia pure sotto forma di catalogo, o meglio elenco piuttosto disordinato di tutte le stampe presenti in una collezione d'amateur, ci si poteva fare un'idea della quantità enorme e della varietà delle stampe prodotte in Europa sino a quel momento, nonché dell'enorme fortuna di quel genere artistico vecchio di neanche due secoli.

Ma il Baldinucci sembra aver tenuto in conto il libro del de Marolles, da lui mai menzionato, anche per un altro motivo, che non riguarda il Cominciamento, bensì l'altra sua opera assai più importante, apparsa col primo volume nel I68I, le Notizie dei professori da Cimabue in qua (conclusasi postuma nel 1728$)^{7}$, in cui, è ben noto, il racconto storiografico, a partire dal secolo tredicesimo, è scandito in decennali. Infatti nel suo Catalogue, nella seconda pagina dell'Avertissement, il francese annuncia la propria intenzione di scrivere un'opera in ben ventiquattro volumi sugli artisti di tutti i tempi e di tutte le nazioni, incominciando dalle origini seguitando con i greci sino ai suoi giorni, e, quel che qui interessa rilevare, dividendo le ultime parti, ovviamente molto più dense, per Décades. Non può trattarsi di una coincidenza: Baldinucci deve aver letto questa pagina, ed esserne stato influenzato per concepire la partizione per decennali della storia dei suoi professori, tanto più che quell'opera magna il de Marolles, morto nel I68I, non l'aveva mai pubblicata, forse perché soppiantato dalla iniziativa vincente di André Félibien, che lo incalzava sullo stesso tema sin dal $1666^{8}$.

Félibien per l'appunto è l'unico scrittore francese che Baldinucci nomina tra le sue fonti scritte, ma non fra le principali. Ne aveva letto gli Entretiens sur les vies et sur les ouvrages des plus excellens peintres anciens et modernes, almeno i primi dei cinque volumi della prima edizione, apparsi a partire dal $1666^{\circ}$. L'elenco, scandito da qualche commento, di illustri incisori francesi o francesizzati seicenteschi ch'egli dà nel proemio del Cominciamento (Claude Mellan, Michel Lasne, François Rousselet, Abraham Bosse, Robert Nanteuil, Francois Poilly, François Spierre, JeanLouis Roullet, Étienne Baudet, Pierre Louis van Schuppen, Anton Masson, Claudine Stella, Gérard Edelinck), elenco nel quale spiccano per assenza molti non meno illustri, non trova riscontro come tale nelle pagine degli

7. F. Baldinucci, Notizie dei professori del disegno da Cimabue in qua... opera di Filippo Baldinucci, Firenze, editori varii, I68I-I728.

8. Sul de Marolles e la sua collezione, A. Schnapper, Curieux du grand siècle : auvres d'art, Paris, Flammarion, 1994, pp. 247-248, 25I-253.

9. A. FÉlibien, Entretiens sur les vies et sur les ouvrages des plus excellens peintres anciens et modernes, Paris, Marbre Cramoisy, I666-I688. 
Entretiens, dove per altro gli incisori sono nominati alla spicciolata e pochi sono quelli francesi. Ma certamente egli aveva avuto modo di vedere - se come stampe singole o raggruppate non è possibile sapere - le stampe che dal I672 circolavano per l'Europa dando figura ai manufatti artistici, dipinti, sculture, arazzi, medaglie ma anche alle maisons royales e perfino alle imprese guerresche, di Luigi XIV, costituendo nell'insieme il cosiddetto Cabinet $d u$ Roi, promosso dal surintendant Colbert quale straordinario strumento di propaganda attraverso le immagini per la gloria di Sua Maestà.

Le stampe dai dipinti (22 italiani su 25 nel Recueil da me consultato a Parigi, Bibliothèque nationale, Aa II, in cui sono inserite anche stampe da marmi antichi), datate dal I672 al I68I, presentano didascalie in francese $\mathrm{e}$ in latino dettate da Félibien (che è autore dichiarato dell'Avertissement ${ }^{10}$ preposto all'edizione dei Tableaux du Cabinet du Roi... I677) "I , fra le quali anche stampe firmate proprio da alcuni di quegli incisori francesi o francesizzati, nominati nel Proemio baldinucciano del i686, Baudet, Masson, Mellan, Rousselet, Edelinck; artefici scelti come il fior fiore di quell'arte cresciuta ultimamente in Francia all'ombra dell'Académie, apice con pochi riscontri altrove del progresso dell'arte dell'intaglio che il nostro per sommi capi intendeva narrare, e per i quali almeno una segnalazione era dovuta. Quanto agli altri due francesi citati nella rassegna di protagonisti offerta nel Proemio dei quali poi scriverà le biografie, Nanteuil e Spierre, che non sono rappresentati nei Tableaux du Roi, imput e informazioni Baldinucci le avrà in modo più diretto e puntuale dall'incisore Domenico Tempesti fiorentino rientrato in patria nel 1678 dopo un apprendistato durato due anni a Parigi presso Nanteuil, il quale certamente gli avrà parlato anche dei pupilli del celebre incisore ritrattista, Jean-Louis Roullet, il van Schuppen, l'Edelinck; infine, nel caso dello Spierre, addirittura per frequentazioni personali avvenute a Roma e a Firenze, nell'ambito di Pietro da Cortona e del Bernini.

Di fatto dal Félibien degli Entretiens il nostro trae trascurabili informazioni. Ma nell'Apologia, il capitolo con il quale nel I68I apre il primo

Io. A. Félibien, Tableaux du Cabinet du Roy. Statues et bustes antiques des Maisons Royales, Paris, Imprimerie royale, 1677.

II. Per la ricostruzione della genesi dell'impresa, la composizione dei vari insiemi tematici e per il ruolo di Félibien, M. Grivel, «Ouvrages, volumes ou recueils? La constitution du recueil du Cabinet du Roi», in À l'origine du livre d'art (Actes du colloque de l'Institut néerlandais, Paris 2006), Milano, 20I0, pp. 65-79, in particolare per le stampe da dipinti, p. 69. Per commento e illustrazioni di alcune di tali stampe, E. Borea, Lo specchio dell'arte italiana. Stampe in cinque secoli, Pisa, Edizioni della Scuola Normale Superiore, 2009, cap. XXXI; per il Cominciamento, ivi, cap. XXXII; e passim per situazioni e bibliografie relative agli incisori sopra menzionati. 
volume delle Notizie $^{12}$, Baldinucci chiamava in causa l'autorevole francese con la trascrizione in italiano di un'intera pagina del primo Entretien, a sostegno della tesi da lui propugnata, in linea col Vasari e dal Félibien condivisa, che spetti indiscutibilmente a Cimabue, e quindi a Firenze, il merito di aver rinnovato la pittura. Al fiorentino questo importava più di tutto, per contrapporsi al bolognese Carlo Cesare Malvasia ${ }^{13}$ (Felsina Pittrice, I678. I, pp. I-I3) feroce critico del Vasari per la sua difesa del primato della toscanità.

I2. F. Baldinucci, Notizie dei professori del disegno da Cimabue in qua, I, Milano, Società tipografica dei classici italiani, I8II, pp. 62-63.

13. C. C. Malvasia, Felsina pittrice vite de’ pittori bolognesi alla Maestà Christianissima di Luigi XIIII re di Francia e di Navarra il sempre vittorioso consagrata dal co. Carlo Cesare Malvasia Fra Gelati l'Ascoso. Divisa in duoi tomi; con indici in fine copiosissimi, Bologna, Barbieri, MDCLXXVIII. 